\title{
Vivre dans les grands ensembles (extrait)
}

Living in Housing Estates (extract)

\section{René Kaës}

\section{OpenEdition}

\section{Journals}

Édition électronique

URL : http://journals.openedition.org/crau/314

DOI : $10.4000 /$ crau.314

ISSN : 2547-5746

\section{Éditeur}

Éditions du patrimoine

\section{Édition imprimée}

Date de publication : 1 décembre 2009

Pagination : 161-172

ISBN : 978-2-85822-944-4

ISSN : 1296-4077

Référence électronique

René Kaës, "Vivre dans les grands ensembles (extrait) », Les Cahiers de la recherche architecturale et urbaine [En ligne], 24/25 | 2009, mis en ligne le 01 septembre 2017, consulté le 19 avril 2019. URL http://journals.openedition.org/crau/314 ; DOI : 10.4000/crau.314 
"Le chercheur ne peut pas, ne doit pas se désintéresser de l'utilisation qui est faite de sa recherche ». Telle est la certitude exprimée par PaulHenry Chombart de Lauwe dans la préface qu'il rédige pour I'ouvrage de René Kaës aux Éditions ouvrières. En 1963, Vivre dans les grands ensembles est un livre à la fois précoce et précurseur. C'est même un des tout premiers livres parus sur la question des grands ensembles, ce sujet de société qui suscite à l'époque déjà l'inquiétude mais pas encore la douleur. Dans sa présentation, Chombart de Lauwe insiste en soulignant que "les tâches sont immenses [et que] le travail interdisciplinaire entre architectes, ingénieurs, urbanistes et représentants des Sciences Humaines ne fait que commencer ». Avec le recul du temps, on perçoit que l'ouvrage lumineux de Kaës n'a peut-être pas été bien lu par ceux qui devaient assumer ce commencement.

\section{Vivre \\ dans les grands ensembles*}

RENÉ KA ËS

« Pour vivre heureux, vivons cachés »?

Beaucoup de ceux qui n'habitent pas un grand ensemble pourraient être tentés de rectifier: survivre dans les grands ensembles. Les raisons qu'ils invoqueraient pour confondre l'optimiste seraient celles-ci : il n'est pas possible de vivre humainement lorsque la liberté concrète est paralysée par un double et rigoureux déterminisme: le poids des structures urbanistiques et architecturales impose un style de vie débilitant, le déséquilibre des structures démographiques et sociales compose un milieu psychosocial néfaste. La vie quotidienne, c'est l'ennui, c'est l'enfer, ce sont les autres trop présents, c'est la solitude dans la foule. Bien des exemples pris parmi les premières réalisations appuieraient leur argumentation.

L'avis des habitants des grands ensembles serait vraisemblablement plus nuancé; il varierait selon la situation de la ville nouvelle ou du nouveau quartier qu'ils habitent par rapport aux lieux de travail et de culture, selon la fréquence des moyens de communication, selon la qualité de leur logement antérieur, leur statut socioprofessionnel, la taille de leur famille, l'aménagement de leur logement actuel,

\footnotetext{
* Publié à la fin de l'année 1963 aux Éditions ouvrières dans la collection "Vivre son temps", Vivre dans les grands ensembles est le troisième livre de René Kaës qui est à l'époque psycho-sociologue à I'Institut du Travail de Strasbourg. L'extrait publié par les Cahiers reprend ici les premières lignes (p. 68-69) et les dernières pages (pp. 134-145) du chapitre III de l'ouvrage. La rédaction remercie l'auteur de l'avoir autorisée à reproduire cet extrait.
} 
l'équipement collectif de l'ensemble. Les préjugés, d'autant plus tenaces que ceux qui les adoptent sont moins impliqués personnellement dans une aventure, sont réduits par l'expérience. Que la vie quotidienne d'un style nouveau qui est en train de s'élaborer dans les grands ensembles soit largement tributaire du béton et de la pyramide des âges, tous les habitants l'éprouvent vivement chaque jour. Mais avant de prendre parti, il convient sans doute d'examiner attentivement comment on vit dans les grands ensembles. Des enquêtes ont été menées: elles enseignent que, bien souvent, les nouveaux venus s'estiment plus heureux depuis qu'ils vivent dans leur nouveau cadre résidentiel ; les témoignages d'animateurs sociaux, politiques, culturels affirment que la vie collective y est à la fois plus spontanée et rendue plus difficile par l'absence d'équipements collectifs. Des faits semblent contredire ces informations : des suicides, un état de nervosité aggravé et dû à l'isolement, à l'éloignement, à l'absence d'insonorisation des immeubles, au sous-équipement, une pathologie nouvelle sont là, tragiques ponctuations d'une vie nouvelle qui se cherche. Tous ces faits, tous ces témoignages alimentent une mythologie (Métropolis contre Utopie), renforcent des préjugés; ils invitent aussi à poser la question : est-il possible de vivre dans les grands ensembles? [...]

\section{Le grand ensemble malade}

"Voici venu le temps des casernes civiles... »

(M. Pialat, L'Amour existe, court métrage)

Le bon sens s'étonne et se scandalise que l'on puisse déjà parler d'une pathologie des grands ensembles. II lui semble inconcevable que des mesures destinées en partie à éliminer les effets du taudis soient, en certains cas, responsables de troubles et dérèglements psychiques et nerveux. Et pourtant, l'expérience de médecins et de psychologues conduit à poser un problème grave; sans doute, les observations recueillies sont-elles encore trop peu fréquentes et non organisées dans un plan de recherche systématique pour qu'elles fournissent des éléments de diagnostic certains. Les faits sont là, cependant, qu'il s'agit de connaître. Les études du Dr Hazeman, des professeurs Heuyer et Lafon, de Mme M.-J. Chombart de Lauwe, de M. Michaud et d'autres chercheurs ont depuis longtemps mis en évidence le lien étroit qui unit la délinquance aux conditions défectueuses de l'habitat. C'est ainsi qu'une enquête menée, au cours de ces dernières années à Grenoble, par MM. Lafon et Michaud a pu confirmer les résultats de travaux américains (1953), portant sur l'agglomération new-yorkaise et établir que, dans $95 \%$ des cas pathologiques et $81 \%$ des cas de délinquance, la qualité de l'habitat est insuffisante. Poursuivant leurs recherches, les enquêteurs français ont observé que les zones de la délinquance juvénile se situent aussi bien dans les cités nouvelles que dans les îlots insalubres, bien que le peuplement soit trois fois plus dense dans ces derniers. Bien plus que le taudis, I'entassement, la vie sociale close (contamination) semblent être de puissants déterminants de la délinquance. Les conclusions de leur rapport ${ }^{27}$ insistent sur la nécessité de respecter l'intimité des familles et d'éviter à la fois l'isolement des jeunes (terrains vagues) et une vie sociale en circuit fermé. Le professeur Lafont observe, d'autre part, que certains

27. On pourra en connaître l'essentiel dans la livraison de mai 1955 d'Études et Documents. 
facteurs de délinquance juvénile sont réunis dès la création des grands ensembles: la promiscuité et la densité d'occupation dans les appartements et dans les espaces communs (cages d'escaliers, cours d'immeubles). Les études de P.H. et M.-J. Chombart de Lauwe ont montré qu'un logement surpeuplé entraîne une augmentation des cris de la mère inversement proportionnelle à la surface de celui-ci. Ils ont montré aussi l'isolement des jeunes minoritaires parmi la masse des adultes et des petits enfants (cet isolement les conduit à vivre en circuit fermé, repliés sur eux-mêmes dans une attitude défensive, et à rechercher des occasions de se réunir hors des zones de circulation générale pour échapper au contrôle social: caves, terrains vagues, greniers); l'homogénéité démographique et sociologique, favorable aux tensions psychosociales qui ne se résolvent pas à cause de la difficile appartenance des habitants à plusieurs circuits de relations sociales; enfin, l'absence de tout équipement susceptible, dans certaines conditions d'accueil et d'encadrement, d'attirer les groupes spontanés de jeunes adolescents.

La suppression du milieu habituel actualise les tendances asociales contenues jusqu'alors, et cette émergence est d'autant plus facile que les conditions d'accueil et de vie quotidienne sont néfastes à une adaptation sociale. C'est en ces termes que se comprennent les difficultés d'adaptation des adolescents et les déséquilibres caractériels des adultes (particulièrement des mères) dans les grands ensembles surpeuplés ou mal équipés.

La délinquance juvénile urbaine, selon les observateurs spécialisés, s'accroît actuellement avec intensité. «Plusieurs enquêtes effectuées en Grande-Bretagne, rapporte M. G. Houist devant le Conseil Économique et Social, ont montré que le taux de la délinquance est parfois plus élevé dans les nouvelles cités que dans les vieux tandis. " Tout en conservant une attitude d'alerte, il faut toutefois se garder de généraliser ces observations à tous les grands ensembles.

Avant d'arriver à des formes critiques de la pathologie, de nombreux troubles peuvent être notés, qui apparaissent, certes, aussi dans d'autres cadres résidentiels (villes anciennes, groupes d'immeubles confortablement aménagés) mais avec une fréquence et une intensité moindres. D'une manière générale, la vie en grands ensembles est une cause aggravante de la pathogénie. Quelles sont les principales formes de manifestations pathologiques rencontrées? Tout d'abord, une plus grande fragilité nerveuse, pouvant entraîner des troubles bénins du sommeil, une impression permanente de lassitude ou une instabilité psychomotrice, surtout chez les enfants qui semblent éprouver de plus grandes difficultés à fixer leur attention que ceux des quartiers urbains traditionnels. Les adultes ne sont pas à l'abri de cette tendance à l'instabilité. Le Dr Hazeman ${ }^{28}$ observe que «le manque de place pour le groupe tout entier favorise le besoin d'évasion par la vitesse qui cherche à multiplier l'espace ».

Les adultes, dont le psychisme et le système nerveux sont moins plastiques, vont quelquefois jusqu'à adopter un comportement névrotique : angoisse, phobies, peur de traverser les rues, les places, crainte d'aller en ville. Des formes aiguës de l'angoisse ont pu, en quelques occasions qui ont vivement frappé l'opinion, entraîner des tentatives de suicide ou le suicide. Certains journaux n'ont pas manqué d'amplifier ces événements et ont ainsi

28. Voir, par exemple, R. H. Hazeman:

"La liberté concrète, condition de la santé

physique et mentale ", Revue d'hygiène

et de médecine sociale, t. VII, n 1, 1959. 


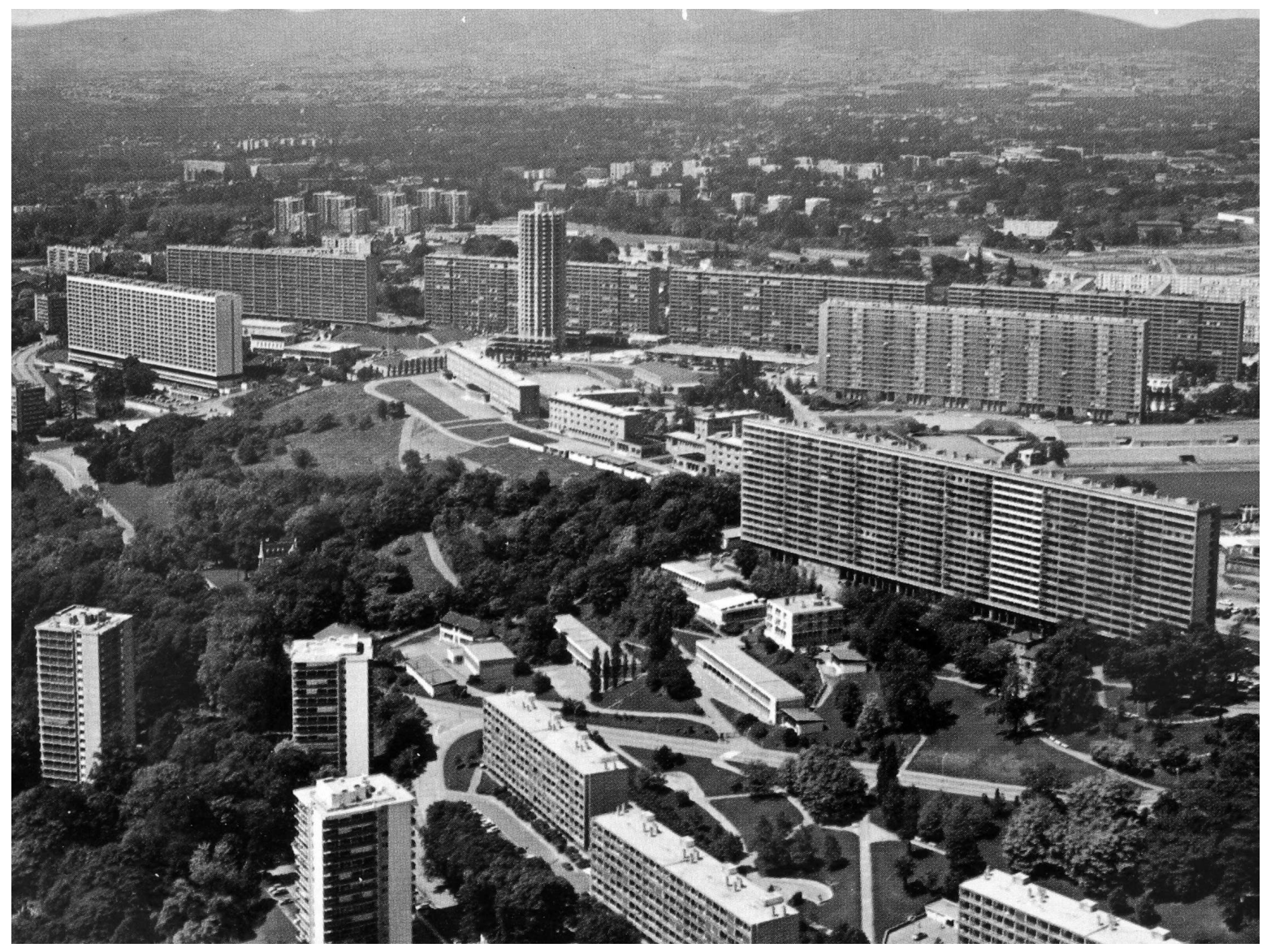

Quartier La Duchère,

Lyon.

Coll. part. F. Bunuel. 


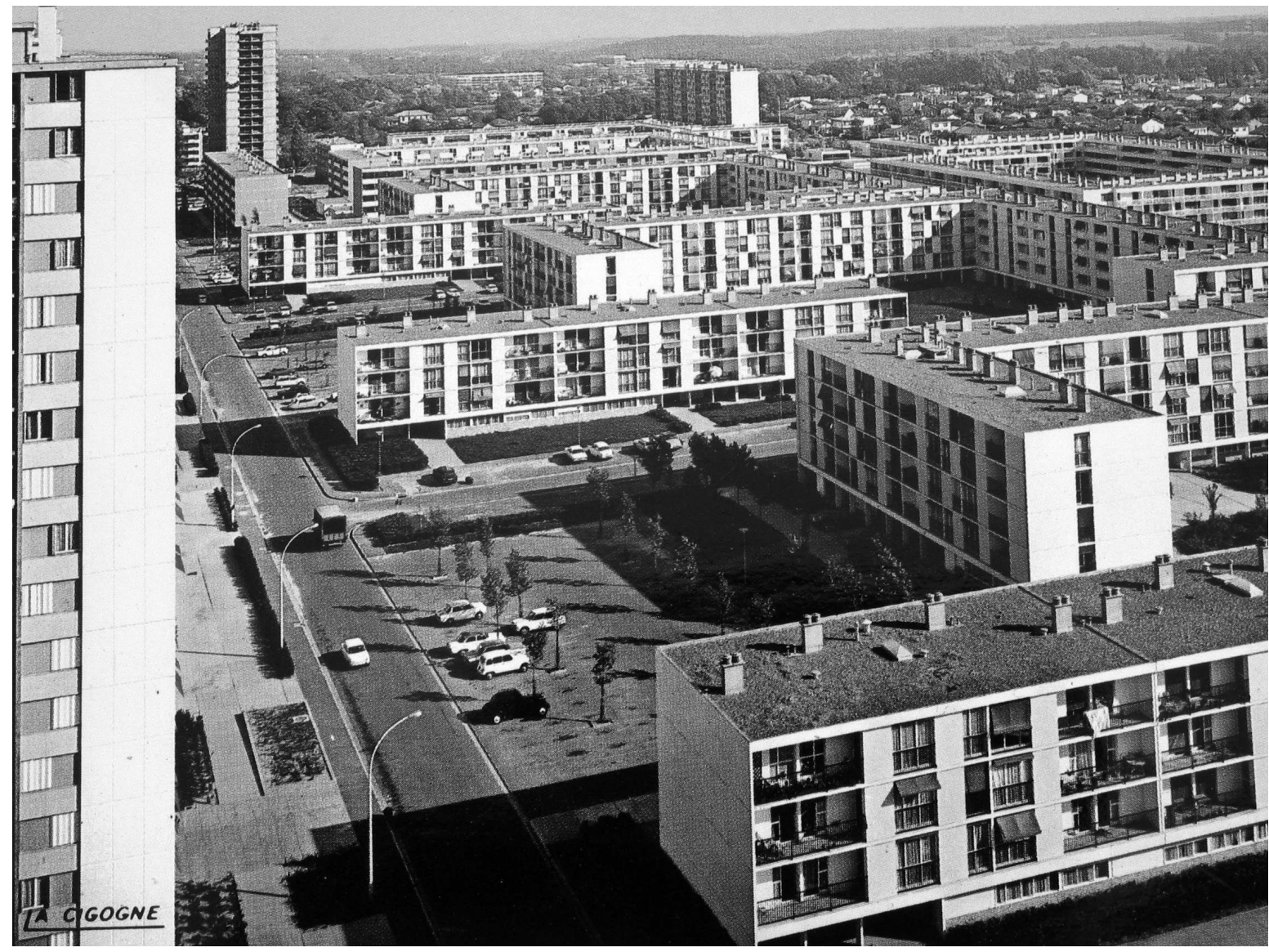

Cité Palmer,

Cenon.

Coll. part. F. Bunuel. 
fortement contribué à la structuration du mythe du grand ensemble mortel.

Mais quiconque vit quelques semaines dans un grand ensemble ne manque pas d'être frappé par le comportement de certaines ménagères dont l'essentiel du temps est occupé à faire et refaire le ménage, frotter et refrotter vitres et parquets, laver plusieurs fois par jour cuisine et salle de bains. Le caractère inadapté d'un tel comportement, que ne justifie jamais une particulière densité de poussière, traduit une structure névrotique obsessionnelle dont l'angoisse est ici encore le moteur: ces femmes, qui peuvent par ailleurs être très occupées, se donnent un surcroît de travail pour éviter ainsi d'être un instant seules avec leur liberté, dont elles ne sauraient alors que faire.

Dans un nouvel ensemble de Strasbourg, nous observons un phénomène curieux: bien qu'il ne porte en soi aucun signe de grave dérèglement pathologique, il nous semble cependant caractériser un type de comportement particulier à la vie en grand ensemble. Dans la plupart des cages d'escaliers, devant les portes d'entrée des appartements, le visiteur pourra se demander pourquoi toutes les chaussures de la famille sont exposées, comme si elles attendaient en permanence de recevoir on ne sait quel cadeau providentiel... En réalité, le cadeau - le sacrifice - est offert aux dieux domestiques de la propreté, de la cire et du balai ${ }^{29}$ !

Ces traits de comportement obsessionnel peuvent se conjuguer avec d'autres formes névrotiques plus ou moins graves: manifestations dépressives, états d'agitation, expriment encore une mauvaise adaptation et une angoisse qui ne trouvent souvent leur délivrance que dans des attitudes de fuite, intoxications pharmaceutiques (tranquillisants, sédatifs), tabagisme, caféisme et, surtout, alcoolisme. De nombreux observateurs, médecins, sociologues, hommes d'action familiale ont dit le paradoxe de cette situation: alors que, par moralisme souvent borné et rigide, le bistrot est éliminé du grand ensemble, un alcoolisme à domicile se développe, et un alcoolisme féminin.

D'autres formes pathologiques expriment ces difficultés multiples de s'adapter à une vie nouvelle : troubles psychosomatiques (états hypertensifs, troubles fonctionnels, manifestations allergiques...) qui peuvent dégénérer en lésions organiques.

Comment rendre compte, prudemment certes, d'une telle pathologie? Nous pouvons la comprendre, d'une manière générale, en termes d'adaptation de l'homme à son milieu. Le passage brutal de la vie rurale, avec ses rythmes et ses types de relations sociales essentiellement déterminés par une tradition, à la vie urbaine et, de plus, à une vie urbaine totalement différente de celle des villes anciennes, est accompagné de frustrations et de blessures psychiques: prenons le cas de l'ouvrier agricole de Corrèze qui se trouve engagé comme manœuvre en Moselle, et logé dans un grand ensemble construit par son entreprise. Il éprouvera, non seulement des difficultés d'adaptation au climat, à son travail, aux trajets, mais, une fois chez lui, il lui sera difficile d'établir de bonnes relations de voisinage ; pour se distraire ou faire ses achats, il lui faudra aller à la grand-ville voisine: ce seront là ses seules sorties, car le grand ensemble n'offre pas de ressources socioculturelles propres: ni bistrots, où d'habitude se rencontrent les individus et les groupes, ni

29. Il est vrai que les habitants alsaciens du nouvel ensemble en question, élevés et élevant leurs enfants très tôt aux premiers rudiments de la propreté (5-6 mois !), sacrifient davantage à ces dieux que les habitants non alsaciens. 
magasins-boutiques où le marchand informe, où les femmes parlent de leurs enfants, ni place publique. Rien de ce qui catalyse la vie sociale spontanée n'existe dans leur nouvelle vie. Ces médiateurs ne sont sans doute pas à reproduire tels quels dans les grands ensembles. Nous constatons seulement ici la dangereuse absence de leur fonction d'intégration sociale. L'urbanisme de la société bureaucratique a fait que la vie des gens des villes est devenue, comme le dit D. Mothé, une vie de Robinson.

Tous les habitants des grands ensembles ne viennent pas de Corrèze pour s'embaucher dans la sidérurgie mosellane. Mais la plupart éprouvent, à des degrés variables, de telles difficultés; la phase d'adaptation varie avec l'âge des nouveaux venus, leur origine géographique, leur état psychique antérieur, et avec l'accueil qui leur est réservé en arrivant, avec l'état d'achèvement et d'équipement du grand ensemble (boue ou macadam, boutiques ambulantes ou centre commercial). Elle varie selon la situation particulière du grand ensemble: c'est ainsi que la situation d'immeubles collectifs construits médiocrement en grand nombre au milieu de petits pavillons individuels, dotés de jardins et d'enclos, ne favorisera guère l'adaptation des habitants du grand ensemble. Nous avons eu, pendant quelques semaines, le spectacle d'habitants nouveaux venus, fascinés et rendus jaloux par le «bonheur » des gens des pavillons (Montigny-lès-Metz; G. Vailland rapporte des observations semblables à propos de Beauregard-de-Poissy). Une seule idée hantait ces inadaptés: partir, et faire construire, "pour soi », sa petite maison.

Chacun ressent douloureusement aussi l'espèce de violation de son intimité familiale dont sont responsables le bruit et le manque de finition des appartements en de nombreux endroits. On a beaucoup parlé de cela dans la presse et cela valait d'être dit: nous avons vu des parents être obligés de déménager d'un nouveau quartier quasi encerclé par des voies de chemin de fer pour éviter à leurs enfants l'aggravation de leurs troubles nerveux; nous avons dû, comme beaucoup d'autres, encourir le risque de nous rendre insupportables à de bons amis voisins pour avoir planté quelques clous dans une étagère un samedi après-midi, ou reçu des amis après 21 heures. "L'obligation de subir, l'impossibilité de réagir, remarque $\mathrm{M}$. Josserand ${ }^{30}$, c'est le type même de la situation irritante, génératrice de traumas. » On ne choisit pas, on est choisi : obligation de subir les voisins que l'on perçoit comme des voyeurs lorsque les habitations largement vitrées sont rapprochées, et toujours comme des écouteurs. Le besoin de silence est nécessaire à l'établissement de bonnes relations sociales, surtout lorsque les formes de sociabilité sont imposées brutalement. Cette notation peut paraître paradoxale à un observateur superficiel: en réalité, le silence n'est pas synonyme d'isolement total et le Dr Hazeman a bien raison de souligner qu' « il y a des limites à tout, que ce soit l'air pur, l'eau pure, l'espace et le silence ».

Il y aurait encore à dire, à propos des effets du gigantisme architectural, sur l'anxiété des habitants, sur la solitude collective (Hazeman) qu'il entraîne; à propos de I'inquiétante absence d'équipements de loisirs et d'éducation pour les enfants. Certes, ceux-ci échappent à la ruelle, au délassement malsain des cours intérieures des vieux immeubles, aux dangers de la circulation, mais peuvent-ils réellement s'ébattre dans les espaces verts, 


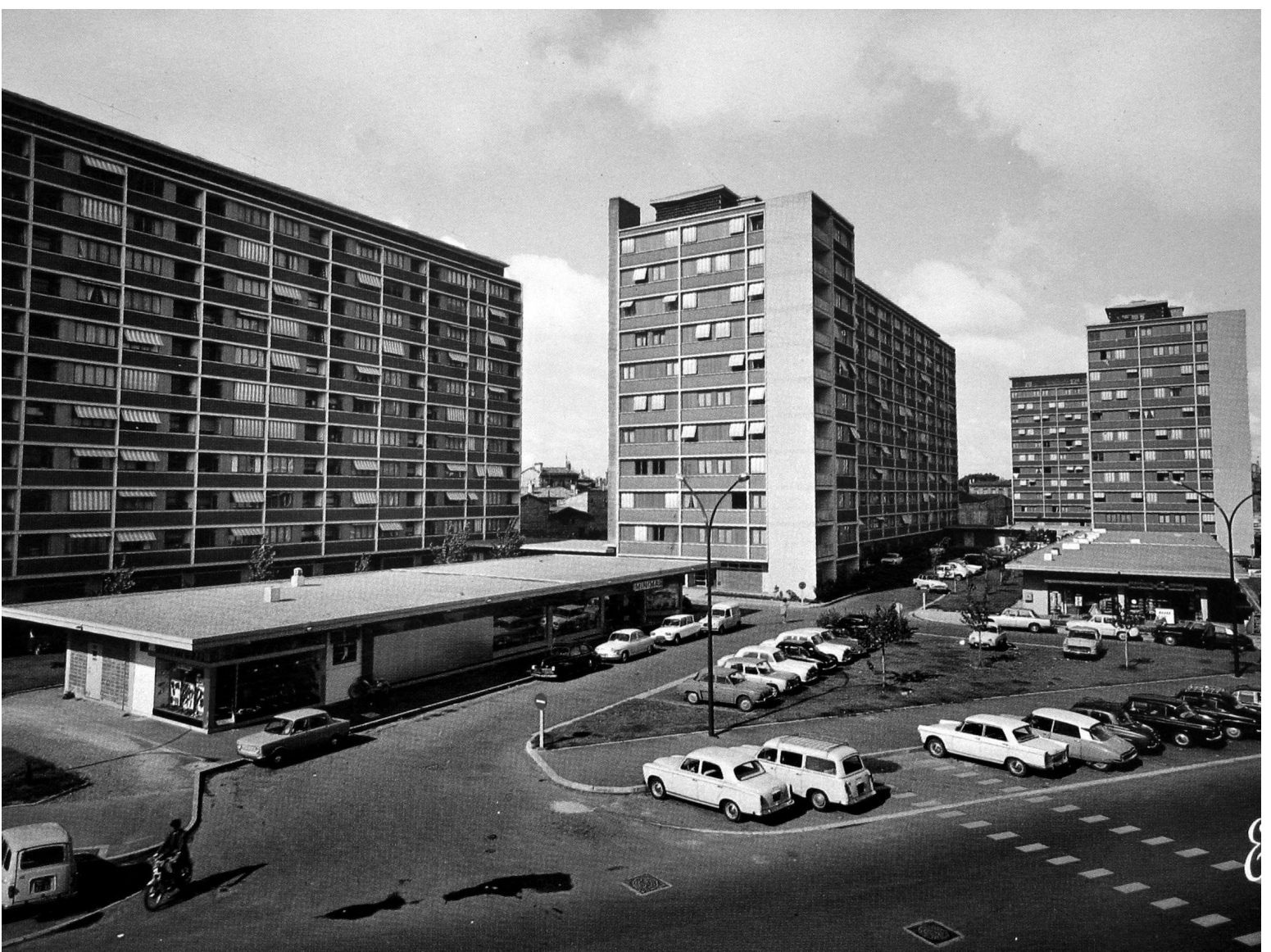

Cité Saint-Jean,

Bordeaux.

Coll. part. F. Bunuel. 


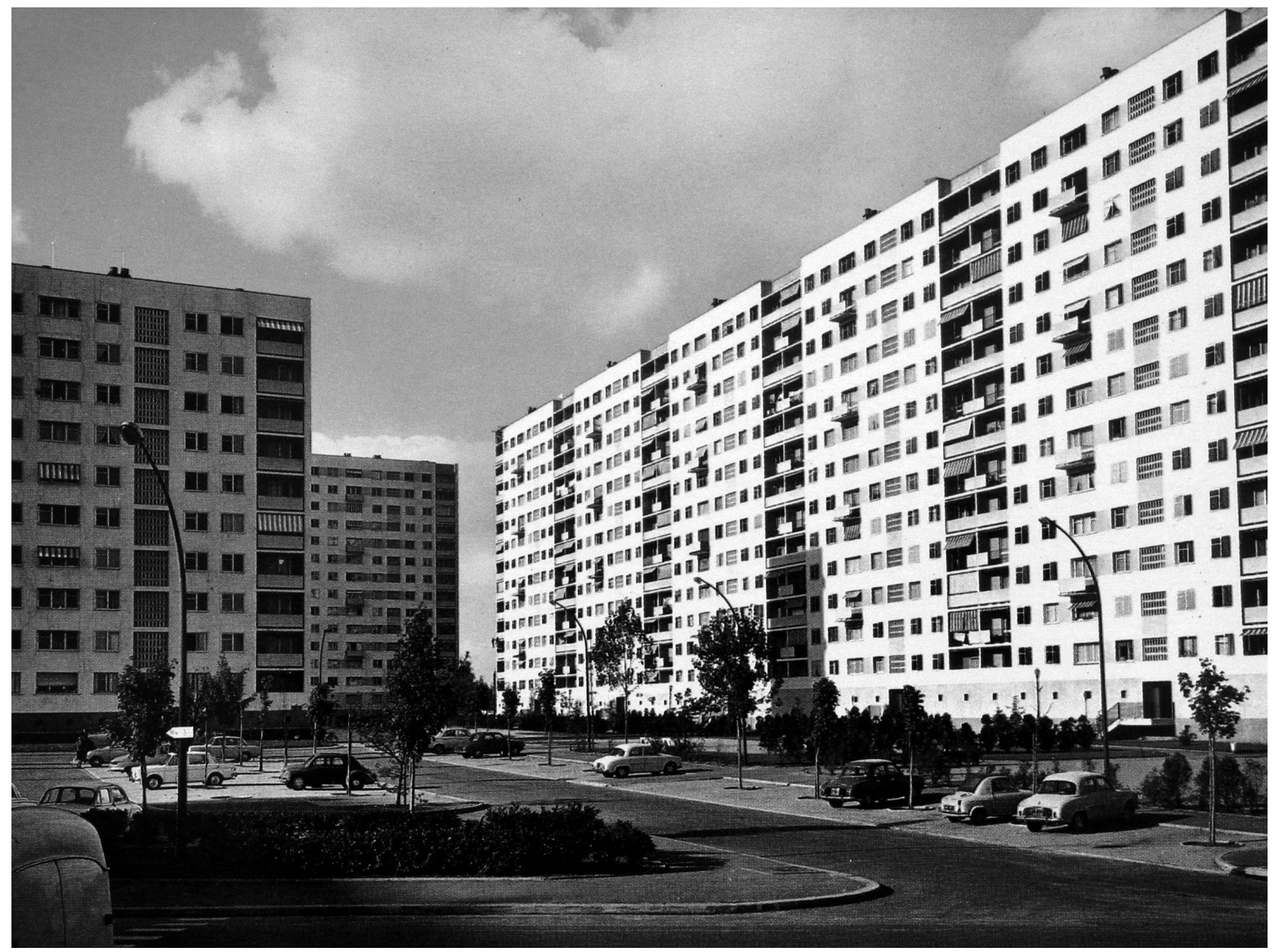

Cité du Grand Parc

Bordeaux.

Coll. part. F. Bunuel*

* Les cartes postales de l'époque, ici reproduites, sont non datées et sont à la base d'un travail de recherche collectif mené dans le cadre du Programme interdisciplinaire de recherche «Art, architecture et paysages »: Frédéric Pousin (dir.), Fabrice Bunuel, Denis Delbaere,
Florent Hébert, Hélène Jannière, Xavier

Michel, « Saisir le paysage urbain: du rôle des publications, figurations architecturales, des pratiques photographiques et cinématographiques dans les décennies 1960-1970». Rapport final septembre 2007, 351 pages. 
aérés et interdits, qui ornent les grands ensembles? Le mérite essentiel du roman de Christiane Rochefort est, sans doute, de saisir tout ce que cette volonté d'hygiène et de bonheur imposé comporte de pesant et d'angoissant. L'homme n'est pas un produit vivant sous cellophane.

Les processus d'adaptation sont essentiellement lents. Ils s'effectuent dans les meilleures conditions, avec du temps.

Mais le temps est-il vraiment un bon guérisseur quand on est contraint de subir et non de choisir? Aucune mesure de replâtrage culturel ou social ne peut être foncièrement efficace.

\section{L'attrait pour les grands ensembles}

Lorsque l'on interroge les habitants de grands ensembles sur ce qu'ils apprécient dans leur vie nouvelle, on ne manque pas d'être frappé par l'abondance des raisons qu'ils donnent pour expliquer l'attrait qu'exerce sur eux cette forme d'habitation. La plupart des familles évoquent le confort supérieur de leur nouvel appartement, mais suggèrent plus ou moins vivement l'amélioration de l'insonorisation et une meilleure finition de détail, une meilleure protection contre le regard des autres (Sarcelles); vient ensuite l'éloge du cadre de résidence, qui offre verdure (surtout pour les Parisiens), air et lumière plus généreusement. L'existence d'un équipement commercial rationnel constitue un bon motif d'être satisfait du grand ensemble: les ménagères y réalisent des gains sur le temps et la fatigue, mais déplorent souvent la cherté des produits par rapport aux commerces traditionnels. Lorsqu'il existe, l'équipement socioculturel est présenté comme un avantage incomparable, surtout s'il concerne les enfants.

L'équipe du Groupe d'Ethnologie Sociale a interrogé les habitants de trois grands ensembles sur leur préférence en matière d'habitation. Près de la moitié des familles choisissent la formule du pavillon individuel et évoquent pour justifier leur choix l'indépendance, la tranquillité, l'espace, la liberté d'ébats pour les enfants, la possibilité de cultiver leur jardin. Un tiers des ménages est favorable à I'habitation collective dans une cité du type de celle qu'il habite: le confort, l'espace, I'air, la liberté des enfants sont les motifs qui reviennent le plus souvent dans la justification des réponses. Un cinquième environ préfère habiter dans un immeuble bien aménagé au centre de la ville, essentiellement pour être à proximité des lieux de travail, de commerce et de loisir. Les réponses varient fortement selon les catégories sociales et selon la situation des cités: les ouvriers choisissent à part égale le pavillon ou le grand ensemble; au contraire, les employés préfèrent, deux fois plus souvent, le pavillon à la cité nouvelle. Les cadres, commerçants, fonctionnaires répartissent leurs réponses conformément à la moyenne générale. Tout aussi intéressante est l'analyse des réponses selon les cités: les résultats enseignent que les habitants choisissent davantage la cité nouvelle lorsque celle dont ils ont l'expérience met à leur disposition de bons moyens de transport et de relations avec le centre de la ville, et lorsque les équipements collectifs sont suffisants et bien conçus.

Ces réponses montrent à quel point, comme le souligne d'ailleurs Chombart de Lauwe, la préférence 
pour un habitat individuel n'a guère de signification tant que les habitants ne savent pas ce qu'ils peuvent attendre de grands ensembles qui leur offriraient un logement adéquat et un équipement satisfaisant. Elles montrent surtout que le grand ensemble n'est pas une formule rejetée d'emblée par ceux qui y habitent.

Peut-être convient-il, en tout cas, de réserver, dans la construction des grands ensembles, une place plus grande aux maisons familiales individuelles; elles sont certainement plus adaptées aux besoins des grandes familles. Cette solution offre, en outre, une possibilité plus étendue de choix parmi les habitants d'un mode d'habitation qui corresponde à leurs aspirations.

\section{Créer de l'histoire}

La ville a besoin de mystère : elle a besoin d'une histoire, d'une mémoire. Elle témoigne d'un passé, c'est-à-dire d'une permanence. Elle lie entre elles les générations qui lui donnent un visage, une physionomie, une âme. On parle de la ville comme d'un être vivant: c'est pourquoi elle a son monument aux morts. Or Saint-Nicolasen-Forêt, Mourenx, Sarcelles-le-Neuf, Farébersviller n'ont pas de monument aux morts : où découvrir, en effet, interroge avec humour G. Lauzun, " les noms à graver sur la pierre, les souvenirs à respecter?... L'Affaire est étrange. Elle trouble la conscience des hommes. Il y a maintenant en France un village (Saint-Nicolas-en-Forêt) sans passé, qui affronte, sans les pouvoir résoudre, les problèmes des villages ordinaires: pas d'aînés morts dans les guerres, pas de bienfaiteurs pour baptiser la rue principale, pas de peintre médaillé. Les gens sont venus de partout : on y parle polonais, italien ou arabe sans qu'une histoire commune soit déjà possible... Le Conseil municipal a décidé d'édifier un monument aux vivants ${ }^{31}$...»

Parce qu'elle n'a pas d'histoire, la ville nouvelle a l'éminent besoin de s'en donner une. Peut-être verronsnous y revivre la fête, la simple fête foraine qui meurt un peu partout, et la fête des groupes et des associations qui vont s'y développer. Le succès de la moindre kermesse laïque ou paroissiale en est déjà un signe prometteur. Mais qui saura organiser la fête où l'on ne s'ennuie pas, dans un grand ensemble où tout conspire contre elle? Qui encouragera et par quels moyens, la naissance de ces groupes spontanés auxquels il faut pourtant une organisation pour subsister? C'est là une des tâches essentielles de l'animation sociale et culturelle dans les grands ensembles.

Mais d'autres conditions sont requises pour que l'histoire se crée. II faut des places pour rassembler les foules; des fontaines et des arbres pour qu'elles s'y sentent à l'aise; des rues où l'on se sente, là, au coude à coude; des lieux familiers où les groupes puissent trouver refuge : le café, la taverne ont cette fonction sociale dans les quartiers « historiques ». Par un triste mélange de puritanisme et de fonctionnalisme excessifs, on a banni les bistrots des grands ensembles, et c'est grand dommage. Quand on ne dispose d'aucun local pour se réunir, le bistrot est encore le seul endroit accueillant pour s'asseoir, boire et discuter: c'est là que naissent les projets collectifs et que se baptisent les amitiés. Mais il vaut sans doute mieux pour l'ordre public que l'alcoolisme à domicile se développe dans certains grands ensembles plutôt que d'exposer une population aux dangers de la spontanéité $^{32}$ ? Il vous prend souvent l'envie d'entrer tout à coup
31. G. Lauzun, Esprit, septembre 1960, p. 1423. À Sarcelles, la SCIC a eu l'heureuse idée de bâtir une sorte de musée de la construction du grand ensemble: cela sert de mémoire collective aux habitants.
32. Un échantillon de café-club (forme policée du bistrot) a été présenté au salon des Arts ménagers 1962 dans le but de ressusciter cet indispensable lieu de réunion et de loisir. 
dans une taverne, au milieu du tumulte, des odeurs et des buveurs, comme après une sinistre randonnée, pour se rassurer d'être ensemble.

Tout aussi navrante est l'absence d'hôtels familiaux et de restaurants. II existe un restaurant à Sarcelles, et les habitants s'en réjouissent; mais, dans la plupart des grands ensembles, ils hésitent à inviter des parents ou des amis parce qu'il leur est difficile de les loger commodément. D'autres boutiques devraient être construites dans les grands quartiers et les villes neuves : des ateliers d'artisans, des magasins-expositions d'artisanat et d'art.

Il manque au grand ensemble des œuvres créées par des artistes pour le plaisir des habitants. Nous nous sommes réjouis, à une récente exposition internationale d'urbanisme, de savoir que les citoyens des grands ensembles de Hambourg, Tapiola (Finlande), Stevenage (Grande-Bretagne), Atakoy (Turquie), Sofia (Grand Ensemble Lénine), Ivrea (Italie) pouvaient jouir chaque jour de statues, de mosaïques, de tours et de fontaines. Dans les groupes scolaires de certains grands ensembles français, un timide effort a été accompli (sans doute parce que la loi de 1951 y oblige). Certaines églises ou chapelles, certains bâtiments publics (Sarcelles, par exemple) sont de bons exemples d'art contemporain vivant dans la cité. Mais n'a-t-on pas parlé de doter les bâtiments publics de moulages d'œuvres éprouvées, garanties haute culture?

Ailleurs, c'est-à-dire presque partout, qu'il voyage dans les grands ensembles de Strasbourg, de Lyon, de Paris, de Lille, Toulouse ou Bordeaux, le visiteur n'a pas l'espoir de découvrir quelque chose de nouveau, d'original, une œuvre qui soit, comme une statue ou une fresque, enracinée dans une histoire et une culture.
Il manque à toutes ces agglomérations nouvelles et à ces quartiers neufs le mystère qui rend la ville complice d'une aventure humaine. Le grand ensemble s'offre tout d'emblée. On ne le découvre pas.

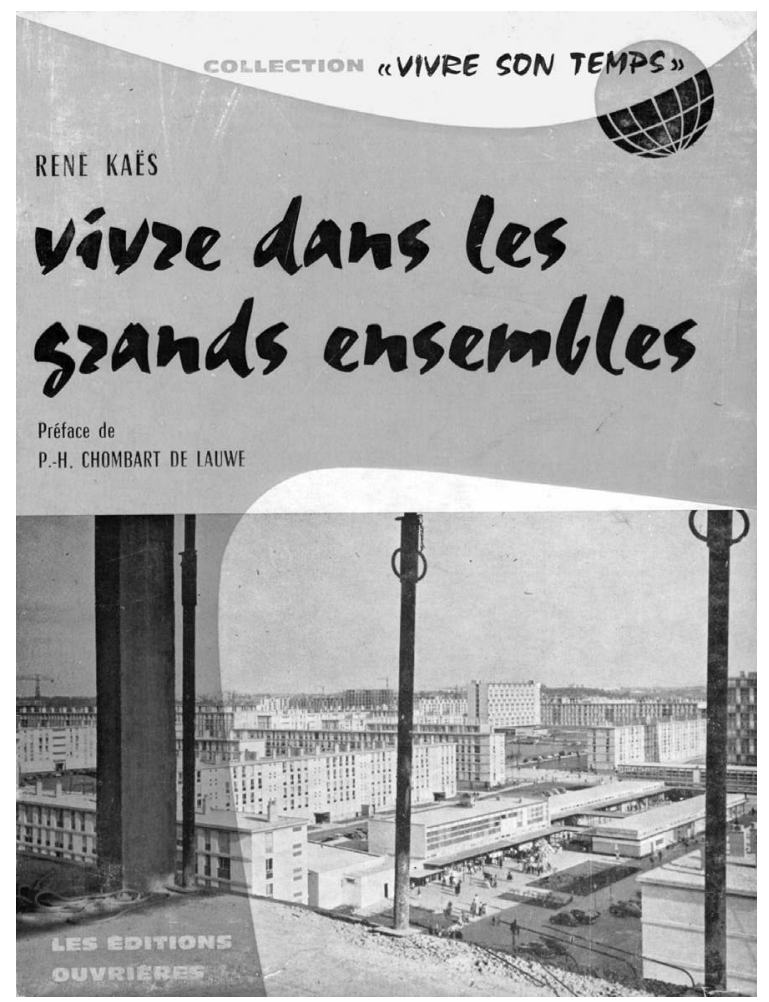

